\title{
A Study on Allergen Knowledge and its Perceived Importance in Food Safety
}

\author{
Kavindya Peiris ${ }^{1}$, Helen Heacock ${ }^{2}$ \\ 1. Lead Author, B.Tech Student, School of Health Sciences, British Columbia Institute of Technology, \\ 3700 Willingdon Ave, Burnaby, BC V5G 3H2 \\ 2. Supervisor, School of Health Sciences, British Columbia Institute of Technology, 3700 Willingdon \\ Ave, Burnaby, BC V5G 3H2
}

\begin{abstract}
Background: Studies have shown that one out of every thirteen Canadians suffers from a significant food allergy, and that 1.2 million Canadians may be affected by food allergies in their lifetime. As food allergies are common, many would assume that allergen safety would be a significant component of public health promotion and food education. This study, on food allergen knowledge, is one step towards addressing the deficit that exists with regards to understanding food allergens in public health.

Method: The study was conducted by surveying Environmental Health Officers (EHOs) nationwide using an online questionnaire. It was distributed via email with the aid of the Environmental Health faculty at British Columbia Institute of Technology (BCIT). The survey was also posted to the BCIT Environmental Health and Canadian Institute of Public Health Inspectors (CIPHI) groups on the online social networking service Facebook. The results helped determine whether opinions, behaviors, and knowledge level regarding food allergens and food allergen safety depended on having a food allergy themselves.
\end{abstract}

Results: The data extracted from the survey was analyzed using the statistical software NCSS. The results of the Allergen Knowledge portion (t-test) concluded that there is no association between the score of the Allergen Knowledge test, and whether or not the participants have a food allergy, inferred by its p-value of 0.268010 . The results of a chi-square test indicated that there is a borderline association between how often EHOs educate restaurant operators on allergen safety, and whether or not they have a food allergy $(\mathrm{p}=0.049)$

Conclusion: The t-test performed concluded that the participant's knowledge regarding food allergens was not dependent on the presence or absence of a food allergy. The second statistical analysis (chisquare test) supported an association between how often EHOs educated restaurant operators on allergen safety, and whether or not they have a food allergy. Health Authorities can use these results to provide a basis for establishing a food allergen training program for EHOs in the future, thereby raising awareness and helping to better manage the presence of food allergens in public health.

Keywords: food allergy, priority allergens, food safety, allergen knowledge, health promotion 


\section{INTRODUCTION}

\section{Food Allergens and Allergies}

In 2010 a nationwide study reported that one in every thirteen Canadians suffer from a significant food allergy (Picard, 2010). Unfortunately, even with such a significant statistic many do not pay much attention to allergens unless they themselves are directly affected by it. This can be attributed to the fact that it is not a strong component of public health programs and therefore is not as enforced by Food Industry Professionals. Food safety education should focus equally on allergen safety as it does the importance of proper food handling practices associated with the preparation, storage, transportation, and serving of food. Everyone should have basic knowledge regarding allergens, especially those working in food service establishments. It is the responsibility of Environmental Health Officers (EHOs), Food Inspectors, and Food Educators to raise awareness and educate the public on the hazards related to allergens, thereby preventing allergen related incidents or fatalities in the future. Currently no allergen safety training programs exist in Canada, but online courses such as ServSafe Allergens, the Food Allergy Toolkit for School Nurses, and How to C.A.R.E for students with Food Allergies are available in the United States (U.S). Additionally, the U.S also offers a program called AllerTrain, which provides allergen training to chefs and foodservice employees regarding safe handling and delivery procedures of meals to diners with food allergies (increase).

The Canadian Food Inspection Agency, CFIA (2014b), defines a food allergen as any protein from nuts (almonds, Brazil nuts, cashews, hazelnuts, macadamia nuts, pecans, pine nuts, which is a seed but considered a tree nut, pistachios, or walnuts), peanuts (a legume), sesame seeds, wheat or triticale, eggs, milk, soybeans, crustaceans, shellfish, fish, or mustard seeds. A food allergy can be defined as an irregular or unexpected response to these food allergens, more specifically the allergic proteins in them, that is triggered by the individual's immune system. The main concern when it comes to allergic proteins is that many foods are identified as allergens despite the fact that there is an absence of relevant supporting data as Lucas and Atkinson's (2008) discuss in their paper "What is a food allergen?” Because of this, it is beneficial to have resources such as Health Canada (2009 \& 2012), the Government of Canada (2012), the CFIA (2014b), as well as other allergy and anaphylaxis related organizations to explain which food allergens should be of concern.

\section{Food Allergies, Intolerances, and Chemical Sensitivities}

A food sensitivity is an adverse reaction to a food that other people can eat without getting sick. Food sensitivities include three broad categories: food allergies, food intolerances, and chemical sensitivities. Whereas food allergies are sensitivities caused by an adverse reaction by one's immune system to certain proteins in food, food intolerances do not affect the individual's immune system and generally causes gastrointestinal issues, while chemical sensitivities are negative reactions to chemicals naturally occurring in or added to foods (Health Canada, 2012). Harvard Health Publications released an Issue in 2011 that extensively covers the differences between food allergies and food intolerances which are described below.

\section{Food Allergy:}

An allergy occurs when "the immune system overreacts to a normally harmless substance" (Harvard Health Publications, 2011, pg. 4). For individuals who have a predisposition for particular food allergies, the immune system responds by producing Immunoglobulin E (IgE) antibodies after the first exposure (Harvard Health Publications, 2011, pg. 4). The IgE antibodies remain in the body attached to mast cells, and if, or when, the individual is re-exposed to that allergen, the allergen binds to IgE which signals the mast cells to release chemicals triggering an allergic response (National Institute of Allergy and Infectious Diseases, 2010). The allergic reaction is a result of histamines being released causing blood vessels to dilate, and leukotrienes being release causing inflammation (Harvard Health Publications, 2011, pg. 4). 


\section{Food Intolerance:}

Food intolerances result from an individual's inability to digest or metabolize food completely (Harvard Health Publication, 2011, pg. 5). Unlike food allergies or chemical sensitivities, this type of food sensitivity requires a larger dose or amount of food to actually cause a reaction. Common intolerances include lactose intolerances where individuals lack the enzyme lactase needed to digest lactose, and impaired complex carbohydrate digestion where individuals who eat large quantities of fermentable carbohydrates such as beans, bran, fruit, sugars, or alcohol sugars may develop symptoms (Health Canada, 2012 \& Harvard Health Publication, 2011, pg. 5). Another example of a common intolerance are gluten sensitivities, characterized by the inability to tolerate gluten, but testing negative for Celiac disease and causing intestinal issues (Harvard Health Publication , 2011, pg.6).

\section{Chemical Sensitivity:}

As previously stated, chemical sensitivities are negative reactions to chemicals naturally occurring in, or added to, foods. It can include annoyance reactions resulting from (Asthma and Allergy Foundation of America, 2005):

- An increased sensitivity to unpleasant odors - Irritational syndromes caused by significant exposure to irritating chemicals that can infiltrate mucosal membranes

- Immune hypersensitivity caused by naturally occurring organic chemicals found in pollen, mold, dust, and animals

- An intoxication syndrome as a result of longterm exposure to harmful chemicals that can cause serious illness or death.

\section{Allergic Reactions}

As mentioned above, an allergic reaction is when an individual's immune system reacts to a particular protein or irritant. When a vulnerable individual comes in contact with an allergen, there is an onset of symptoms leading to reactions ranging from mild to severe.

\section{Common symptoms associated with allergies (Health Canada, 2009):}

- Trouble breathing, speaking, or swallowing
- Itching or swelling in the mouth (lips, throat, tongue), swelling of the eyes

- Vomiting, Diarrhea, and/or, abdominal cramps and pain

- Flushed faces, hives, rash, or eczema

- Tightening of the throat and trouble breathing

- Drop in blood pressure, rapid heartbeat, and/or loss of consciousness

- Anxiousness, distress, faintness, paleness, sense of doom, and/or weakness

\section{Treatment and Prevention}

Currently there is no cure for food allergies, and the only way to prevent them is by avoiding particular foods. A severe allergic reaction can be caused by any of the following: food allergens, insect bites, insect stings, medications, plants, and latex. Reactions can be treated by administering epinephrine which opens up airways reducing breathing difficulties and narrowing blood vessels to prevent low blood pressure which can lead to fainting (Health Canada, 2009).

\section{Priority Allergens}

A priority food allergen is "something that health officials and regulators regard to be frequently associated with food allergies and allergic type reactions" (Culhane, 2012). The Government of Canada and the Canadian Food Inspection Agency identifies ten priority allergens. These include: peanuts, tree nuts, sesame seeds, milk, eggs, seafood (fish, crustaceans, and shellfish), soy, wheat, sulphites (a type of food additive), and most recently mustard (Government of Canada, 2012). The United States, on the other hand, identifies their priority allergens as eight major allergens also known as the Big-8 (University of Nebraska - Lincoln, 2014). These include all the foods in the Canadian list with the exception of sulphites and mustard. Currently there has been a rise in the number of allergen related incidents, which could be contributed to an increase in diagnosis, reporting, or individuals becoming progressively vulnerable throughout the years. The statement that allergens are on the rise is not only supported by Harvard Health Publications (2011) who states that "more than 170 foods have been associated with allergic reactions, but $90 \%$ of all cases involve milk, eggs, peanuts, tree nuts, fish, 
shellfish, wheat, or soy” (p. 4), but also by research conducted by Ben-Shoshan et al (2010) and Soller et al. (2012) who are essentially the same group of scientists presenting several works on allergen prevalence in Canada. Even the Government of Canada emphasizes that food allergens are of great concern and that as many as 1.2 million Canadians may be affected by food allergies, and that the numbers are likely to rise (Government of Canada, 2013). Other professionals state that "food allergies affect an estimated 5 to $6 \%$ of Canadian children and 3 to $4 \%$ of Canadian adults, with a much larger population suffering from food intolerances” (Culhane, 2012).

\section{Statistics}

In 2012 a national study determined that $7 \%$ of Canadians self-report a food allergy (Soller et al., 2012). This Canadian study is a follow-up to a 2010 study conducted by same group of scientists, called "A population-based study on peanut, tree nut, fish, shellfish, and sesame allergy prevalence in Canada” (Ben-Shoshan, 2010). It should be noted that this was the first nationwide study conducted to determine the prevalence of severe food allergies in Canada. The latter study by Soller et al. (2012) determined that the most common allergies seen in children were for peanuts, tree nuts, and milk, while it was shellfish, fruits, and vegetables for adults. Additionally, they found higher rates of self-reporting in households where the main responder had a postsecondary degree or was born in Canada. Unfortunately, these numbers are not indicative of the true population of individuals vulnerable to food allergens, but can be used to encourage individuals to get diagnosed and follow up with suspected allergies. Soller et. al

(2012) also concluded that there is a fault or inadequacy with their results as the prevalence rates depend on individuals who self-reported and therefore it is likely that the rates are a misrepresentation, most likely an overestimation, of the actual prevalence rates of food allergies in Canada. The preceding study by Ben-Shoshan et al. (2010) explained that although many previous studies show an increase in the prevalence of foodinduced allergies and food-related anaphylaxis, all the estimates vary between the studies. This was found to be true even in this research. There were many inconsistencies. The problems that BenShoshan et al. (2010) dealt with included the refusal of participants and physicians to release their results, while some participants did not get tested at all, which provided the researchers with insufficient data being limited by both quantitative and qualitative data. To work around this, BenShoshan et al. (2010) developed a table that not only provided the perceived cases, but the probable cases, and even the number of confirmed cases to allow for a better approximation of the true prevalence exhibited by food allergen vulnerable populations in Canada. The prevalence results are shown in Figure 1. To add to the statistics, Anaphylaxis Canada (2014) referencing Soller et al.'s research, stated that approximately 2.5 million Canadians self-report having at least one food allergy, with one in two Canadians knowing someone with a serious food allergy. Additionally, Canada is said to have 300,000 children under the age of 18 years who have food allergies, with the highest incidence being observed in children under the age of 3 (Anaphylaxis Canada, 2014). The last statistic they shared was that studies show that more than $40 \%$ of Canadians read food labels looking for allergen information (Anaphylaxis Canada, 2014), which is quite low. This is another area of concern. The majority of people who look for allergen information are likely to be those who are susceptible to them, but this does not mean that they will be the only ones to consume that particular food item. It is best to educate yourself and know what is in your food to avoid injury not only to yourself, but to others around you. This is why the implementation of an allergen safety educational or promotional campaign can be beneficial.

Comparing the allergy prevalence in Canada to those in the United States, the rates seem much lower in the United States (Figure 2). This could be indicative of better regulation of allergens through the Food and Drug Administration. According to a United States study by Sampson (2004), approximately 4\% of Americans suffer from food allergies with severe allergic reactions resulting in 30,000 emergency room visits, 2,000 hospitalizations, and 150 deaths per year. It is hard to compare prevalence and incidences between

TABLE II. Prevalence estimates for perceived, probable, and confirmed food allergy

\begin{tabular}{lcccc}
\hline Participants & Peanut & Tree nut & Fish & Sesame \\
\hline Children $(\%)(95 \% \mathrm{CI})$ & & & & \\
Perceived & $1.77(1.21-2.33)$ & $1.73(1.16-2.30)$ & $0.18(0.00-0.36)$ & $0.55(0.21-0.88)$ \\
Probable & $1.68(1.14-2.23)$ & $1.59(1.04-2.14)$ & $0.18(0.00-0.36)$ & $0.50(0.18-0.82)$
\end{tabular}


Canada and the United states as all the studies look at different parameters. The only point that can confidently be made is that in both countries, prevalence is higher than anticipated, and that the prevalence of allergies is continuously rising. Another similarity is that peanuts were the main trigger. This fact is further supported by a study based in the United Kingdom where peanuts, milk, and eggs are the three main triggers related to food allergen-induced reactions (Sicherer, 2011). The U.K based study also showed that there was a rise food allergen prevalence from 3\% to 6\% (Sicherer, 2011), slightly less that the $7 \%$ observed in Canada.

The lack of a complete and thorough data and statistics makes analysis difficult. This statement under the Food and Drug Act. Their role is to ensure appropriate labelling by ensuring that food products and packages list all the allergens present in the ingredients. In the U.S individuals can refer to the Food and Drug Administration and the Food Allergen Labeling and Consumer Protection Act (FALCPA) of 2004 for laws and requirements regarding food allergens (U.S. Food and Drug Administration, 2013). These legislations are much more comprehensive and provide more information regarding the handling of food allergens within the food industry. The FALCPA provides those interested with background information on allergens and allergic reactions, allergic foods of concern, current findings on major food allergens, allergen and allergy

Table II-2. Allergy Prevalence in the United States

\begin{tabular}{|c|c|c|c|c|c|c|c|c|c|}
\hline \multirow[b]{2}{*}{$\begin{array}{c}\text { Age } \\
\text { Group }\end{array}$} & \multicolumn{9}{|c|}{ Percentage of the Population } \\
\hline & $\begin{array}{c}\text { All } \\
\text { Allergens }\end{array}$ & Milk & Egg & Peanut & $\begin{array}{l}\text { Tree } \\
\text { nuts }\end{array}$ & Fish & Shellfish $^{\mathrm{a}}$ & Wheat & Soy \\
\hline Children & 6.0 & 2.5 & 1.3 & 0.8 & 0.2 & 0.1 & 0.0 & $\mathrm{UNK}^{\mathrm{b}}$ & 0.2 \\
\hline Adults & 3.7 & 0.3 & 0.2 & 0.6 & 0.5 & 0.4 & 2.0 & $\mathrm{UNK}^{\mathrm{b}}$ & $\mathrm{UNK}^{\mathrm{b}}$ \\
\hline
\end{tabular}

is reaff Figure 2. Allergy Prevalence of the 8 Major Food Allergens in the United-States. Retrieved from 1

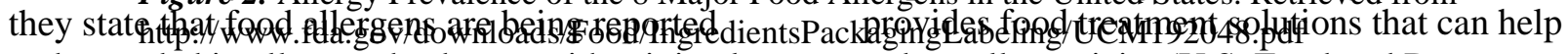

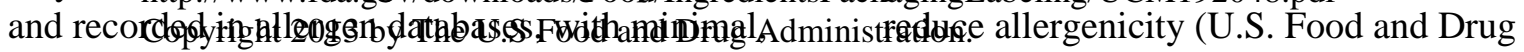
or in some cases, apparently no published justification. Several sources present varying prevalence numbers and many do not provide any background to their findings. This is one of the main issues that need to be addressed. Food industry professionals can use statistics to analyze the current national, provincial, or regional status of food allergen prevalence, allowing them to develop proper educational and promotional tools. These tools can be used by food industry professionals to educate, persuade, and potentially enforce appropriate actions to prevent foodinduced illnesses.

\section{Legislation}

Although there is some legislation in Canada regarding allergens, it is quite vague and not very comprehensive, which is why actions such as enforcement can be difficult. Allergens are regulated by Health Canada through the CFIA Administration, 2013). Furthermore, a strong aspect about the FALCPA is that the legislation can also act as a resourceful guidance document that provides recommendations and references on current research conducted on various food allergens, to help determine their prevalence nationally. Although enforcement of food allergen safety is less of a focus in public health and promotion in Canada, compared to areas such as temperature abuse, it continues to slowly gain recognition. For example, Culhane (2012) mentions that they want to change the way of labelling in the future to make sure that all food labels in Canada list the priority food allergen by its official name adjacent to the ingredient. This is done to make reading labels easy by helping people better recognize foods that they should be concerned with. For example ingredients that show flour, ovalbumin, and seasoning, will now read: flour (wheat), ovalbumin (egg), and seasoning (mustard) (Culhane, 2012). The purpose 
of updating old legislation and establishing new legislation is to stay current. It is important to provide the public with relevant information based on changes observed within the population to help control, reduce, or eliminate the health hazard, ultimately providing the public with a safer environment. In situations where an undeclared allergen is able to cause a potentially serious health hazard, the CFIA is responsible for ensuring that the product is recalled from retail and commercial facilities, which is then followed up by issuing a public warning (Government of Canada, 2012). In addition to the Food and Drug Act, the CFIA also has several advisories regarding allergens in food which are readily available to industry professionals as well as consumers on their official website.

\section{Food Recall Concerns}

Out of the average 250 food recall incidents a year, the number one reason for food recalls in Canada are undeclared allergens, defined as a food item that contains ingredients such as peanuts, milk, or eggs that aren't identified on the label and have the potential cause an allergic reaction (CFIA, 2013). The fact that food allergens are being highlighted is both reassuring and problematic as we are led to believe that CFIA is aware of the presence of allergens in foods, but the high numbers remain discomforting. Recalls can be a big loss to the company from a financial and consumer perspective. Additionally, the frequency and extent of the recall can affect business and their reputation in the community can change for the better. As mentioned in the previous section, in a case of a mislabeled or undeclared allergen, it is the job of the CFIA to initiate a recall, notify the public of the current situation, and confirm that the product has actually been recalled and taken out of the market.

\section{Relation to Public Health and the Proposed Study}

Due to the increasing prevalence of allergies, it is important to identify allergens of concern, and as Lucas and Atkinson (2008) explain, industry professionals such as allergists, scientists, the food industry itself, and food regulators like EHOs and Food Inspectors should make it a top priority.
Because of the rise in the number of allergeninduced illnesses and the number of annual recalls, certain advancements and developments in public health promotion need to be implemented. The Government of Canada has started making changes. They have improved access to allergen information by setting up an email alert for allergen recalls to everyone subscribed to CFIA. This new program is a way that CFIA can protect the health and safety of Canadians by allowing the public to make informed food choices (CFIA, 2011).

The purpose of this research study was to determine the level of knowledge Environmental Health Officers have regarding food allergens, and whether it has an impact on how much of a focus it is in public health. Online surveys were distributed to Canadian EHOs early in 2015 including questions like their age, when they started working as an EHO, their region of employment, whether they themselves or others they know have allergies, particular behaviors and opinions regarding food allergen importance, and ending with a knowledge testing section. This information was then analyzed to determine whether there is an association between the EHOs background and their knowledge regarding food allergens. Responses regarding how much of a priority food allergen safety education is to EHOs were also closely analyzed.

\section{MATERIALS AND METHODS}

\section{Description of Materials Used}

To conduct and complete this survey, access to a computer, the internet, and Google Docs were required. The survey was attached to a consent form introducing the researcher, addressing the purpose of the study, and inviting participants to complete the questionnaire. The consent form also addressed any confidentiality concerns. The results from Google Form (the survey) were automatically recorded onto Google Spreadsheet (online version of Microsoft Excel). The data was then exported into NCSS 9, a computer program for statistical data analysis (Hintz, 2013), to analyze and interpret the results. 


\section{Description of Methods Used}

The survey, which was generated online, using Google Docs, was created by making a Google Form as previously mentioned. The survey was initially posted onto the BCIT Environmental Health and Canadian Institute of Public Health Inspectors (CIPHI) groups on the online social networking service Facebook. It was then emailed to the directors of British Columbia's regional health authorities. Lastly, the president of CIPHI helped distribute the survey to EHOs nationally.

Self-administered surveys or questionnaires by email such as this one are often found to not only be time and cost effective, but receive a higher response rate compared to other methods (Heacock \& Sidhu, 2014). With programs such as Google Docs, conducting a survey does not require extra fees, and since many of its functions are automated, data entry and analysis take significantly less time. Responses to the survey can automatically be collected in Google Spreadsheet, which is the Google Docs version of Microsoft Excel. After this step data may be downloaded directly onto Microsoft Excel for further analysis by selecting the "File" tab on the menu bar, followed by "Download as," and ending with "Microsoft Excel." Real-time response information, and breakdown charts were available with Google Forms allowing the researcher to keep track of how many responses were received, and to get a general idea of the response quality and statistics. The survey results were analyzed in NCSS 9. A t-test compared scores for "Allergen Knowledge," between those with allergies and those without. A chi-square test compared the "Background Information" responses with the responses in the "Opinions and Behaviors" section (refer to Appendix A.2 for the survey). The results obtained were interpreted to help make conclusions regarding relevant associations between the categories of concern. Overall, when conducting a survey, especially one conducted via email, the methods are quite simple and standardized. A well organized, worded, and formatted survey can not only be easier for the researcher to analyze, but easier for participants to provide the most meaningful and valid answers.
Lastly, the study participants were not informed on the fact that they were being scored, nor were they informed on the scoring system. Additionally, the results of their individual scores were not disclosed.

\section{Reliability and Validity of Measures}

In order for an experiment or study to be successful, results should be reliable and valid. Reliability is defined as "the degree to which repeated measurements of the same subject under identical conditions yield consistent results" in the Oxford English Dictionary. To increase the reliability and decrease any errors due to misinterpretation, the same survey was sent to all EHOs in Canada, which asked close-ended questions. Also, to ensure consistency, the survey was only distributed using one method, which was self-administered email.

In statistics, validity is the confirmation that your chosen tool or method is measuring what it is intended to measure. The validity of a survey (the tool or instrument) means that the survey is measuring what it is planned to measure, which in this study are the opinions, behaviors, and knowledge regarding food allergens. Internal validity is "the extent to which the design selected, the instrument used, and the data yielded, permits the accurate conclusions about cause and effect" (Heacock \& Sidhu, 2014). External validity on the other hand, is "the extent to which the results can be extrapolated to a larger, more general population” (Heacock \& Sidhu, 2014). In terms of internal validity, the questions asked made accurate conclusions regarding the presence of food allergies in the participants, and the level of knowledge they have on the subject matter. External validity in this study can only be achieved if a more representative sample is used. Since the survey was only distributed to EHOs, some of the questions cannot be applied to a more general population. Therefore, the results can only be confidently applied to the populations of EHOs in Canada.

\section{Inclusion and Exclusion Criteria}

This particular study was targeted towards EHOs; therefore participants were restricted to practicing 
EHOs. All age groups were welcomed to participate, as were EHOs working regionally in the Lower Mainland, Provincially in BC, or nationally throughout Canada.

\section{Ethical Conditions}

When creating the survey or conducting research, it is important to consider any ethical issues. To address any concerns participants may have, a consent form was attached to the survey asking for their participation, stating the purpose and nature of the study, and reassuring their confidentiality and anonymity. A pilot study was conducted with certified EHOs employed at BCIT to correct for any difficulties encountered, and to make changes to any unclear aspects of the survey. Some survey questions were adapted from a study by Gupta et al. (2009).

\section{RESULTS}

\section{Description of Data}

The data collected was a combination of binary and multichotomous nominal data, multichotomous ordinal data, and numerical data. The nominal data consisted of mostly demographic questions, while the ordinal data involved categories ranking the participant's opinions and behaviors on food safety practices at work and at home. The numerical data was representative of the number of correct answers achieved on the food allergen knowledge portion of the survey.

\section{Description of Statistics}

Results were obtained by 77 different participants of whom 60 (78\%) did not have food allergies, and seventeen (22\%) did. Of the participants who had food allergies, $12 \%$ reported having extremely severe allergic responses (anaphylaxis), 3\% reported having severe symptoms (tingling of the tongue and mouth region, nausea, vomiting, diarrhea), 24\% reported having moderate symptoms (faintness, weakness), and $47 \%$ reported having mild symptoms. Additionally, $68 \%$ of the participants had either a close family member or friend with a food allergy, while $32 \%$ did not. More detailed breakdowns are available in
Figures 3 and 4 representing the results of the Background Information/Demographic section. In should be noted that the location of employment was not taken into consideration in this study as the majority of the respondents (97\%) were employed in the Western Provinces.

Figure 3.

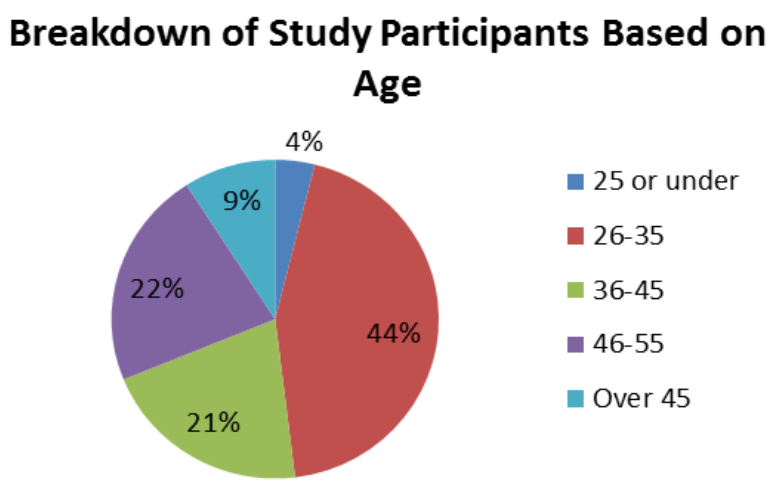

Figure 4.

\section{Breakdown of Study Participants Based on When They Started Working as an EHO}

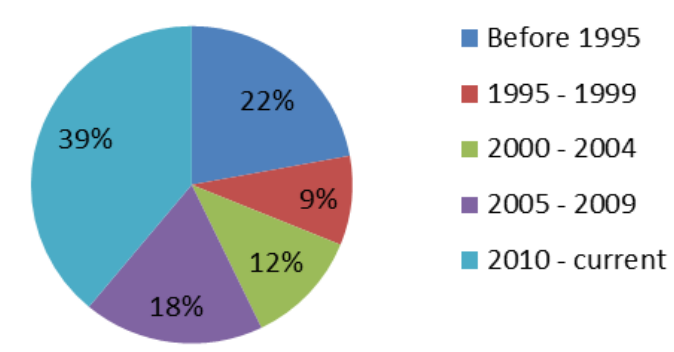

A detailed breakdown of the responses to the question, "How often do you educate food operators on allergy safety?”, and the results of the allergen knowledge section based on the presence or absence of food allergies, are presented in Figure 5 and Table 1 respectively. Participants who had allergies mostly responded to the frequency of education question with "Most of the time," while participants without allergies mostly responded with "Some of the Time" or "A few times.” There were also several participants (21\%) in the study - who were not actively involved in 
the food inspection program. Lastly, for the question "How would you rate your knowledge about food allergens or allergic proteins?” $40 \%$ responded that they were very or quite knowledgeable, $56 \%$ responded that they were moderately or quite knowledgeable, and $4 \%$ responded that they had either limited or no knowledge of the subject matter. association between the presence of a food allergy and the allergen knowledge score, and one chisquare test to determine the association between the presence of a food allergy and how often EHOs educate food operators on allergen safety.

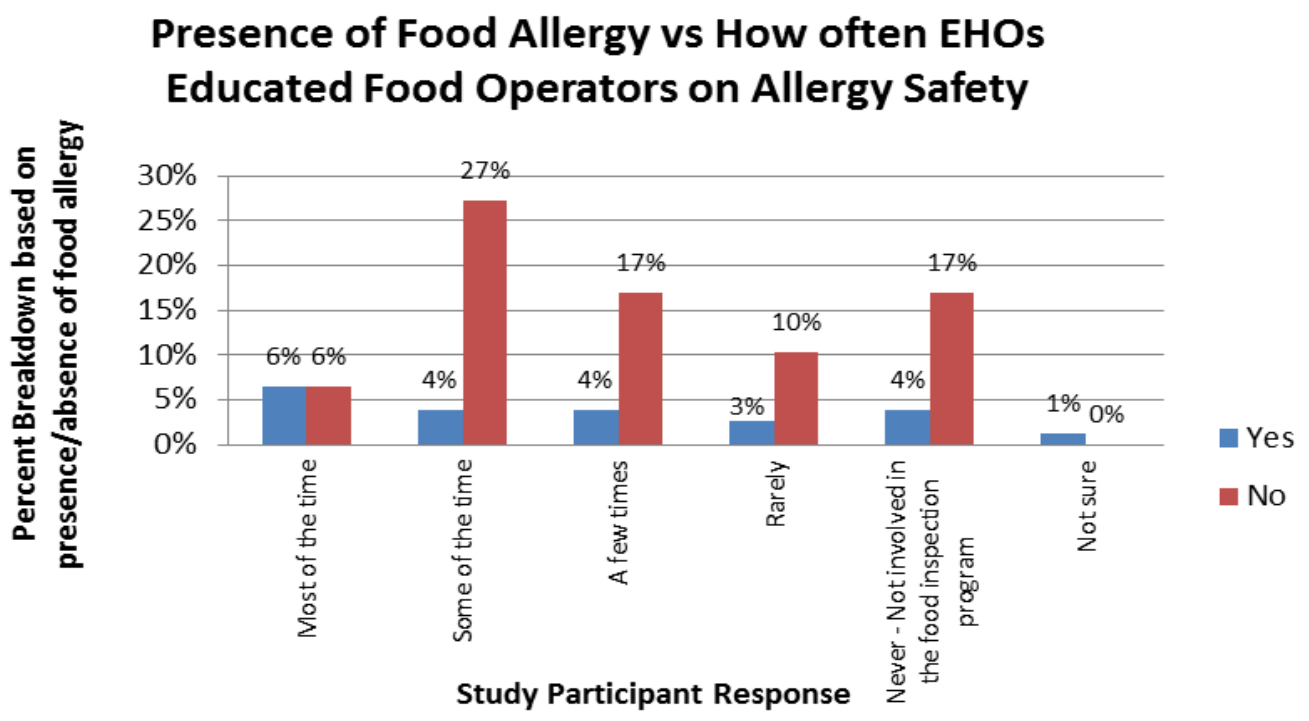

\section{Inferential Statistics}

Figure 5. Excel bar graph showing how often participants with and without food allergies
educated food operators on allergen safety (Microsoft Corporation, 2010)

For this study both a t-test and a chi square test was used to analyze and interpret the data. A t-test is a commonly used statistical method for comparing two distributions (Lee \& Wang, 2013), which in this case is the presence and absence of a food allergy, was chosen to analyze the numerical data obtained from the "Allergen Knowledge" portion of the survey. The chi-square, a nonparametric tool designed to analyze group differences when the dependent variable is measured at a nominal level (McHugh, 2013), was used to interpret the nominal and ordinal data and determine their association. One example of a test that was conducted was the association between the presence of a food allergy, and the amount of allergen safety education participants provided food operators with.

\section{Statistical Package Used}

The statistical software NCSS 9 was used to input data and conduct one t-test to determine the
Table 1. Summary of Results from Food Allergen Knowl

\begin{tabular}{|c|c|}
\hline Test Score Summary & Presence of Food Aller \\
\hline Mean & 7.65 \\
\hline Median & 7 \\
\hline Mode & 7 \\
\hline Standard Deviation & 1.33 \\
\hline Range & 4 \\
\hline Lowest Obtained score & 6 \\
\hline Highest Obtained Score & 10 \\
\hline Count & $17(22 \%)$ \\
\hline
\end{tabular}

\section{Interpretation of Results}

T-test Results: For the t-test, the "Allergen

Knowledge" test scores of 17 participants with food allergies were compared to the score of 60 participants without food allergies, and the following hypotheses were made:

Ho: $\mu 1=\mu 2$

Ha: $\mu 1 \neq \mu 2$

Where $\mu 1=$ Score of Allergen Knowledge test for participants with food allergies. 
$\mu 2=$ Score of Allergen Knowledge test for participants without food allergies.

The NCSS report for the tests of assumptions showed "Cannot reject normality" for the test group with allergies, and "Reject normality" for the test group without allergies. Additionally, both the Equal Variance Tests indicated "Cannot reject equal variances. Taking these results into consideration, the data was deemed to be nonnormal and the next step was to read the Mann Whitney U or Wilcoxon Rank Sum Test for Difference in location. The results showed a probability level (p-value) of 0.268010 . Since the $\mathrm{p}$-value is considerably higher than the 0.05 cut off, it can be deduced that the results are not statistically significant at the $5 \%$ level. Therefore, one cannot reject the Ho. Hence, it can be concluded that there is no difference between the score of the Allergen Knowledge test, and whether or not the participants/EHOs have a food allergy. In other words, those who have a food allergy are not necessarily more likely to score higher on the Allergen Knowledge test than those without a food allergy.

Chi-square Results: For the chi-square test, the responses regarding whether or not the participants

had a food allergy were compared to how often participants or EHOs educated restaurant operator on allergen safety. Responses were collapsed into fewer categories ("Most of the time," "Some of the time," and "A few times/Rarely") and only EHOs actively involved in the food inspection program were analyzed. The following hypotheses were made:

Ho: There is no association between how often EHOs educate restaurant operators on allergen safety and whether or not they have a food allergy.
Ha: There is an association between how often EHOs educate restaurant operators on allergen safety and whether or not they have a food allergy.

As previously mentioned, a chi-square itself is a non-parametric statistical test. The results of the test are presented in contingency tables comparing the Observed frequencies with the Expected frequencies (Heacock \& Sidhu, 2014). Looking at the Tests for Row-Column Independence, the Pearson's Chi-Square gives a chi-square value of 6.0091, 2 degrees of freedom, and a p-value of 0.04956 , which is less than 0.05 , meaning we reject the Ho. Therefore, we can assume that there is an association between how often EHOs educate restaurant operators on allergen safety, and whether or not they have a food allergy.

\section{Alpha and Beta Errors and Power of the Study}

An alpha error occurs when we incorrectly reject the null hypothesis (Ho). In this study the alpha value was set at 0.05 , and based on the results an alpha error could be an issue in the chi-square test as the p-value given was only 0.04956 . Since the $\mathrm{p}$ value was between 0.01 and 0.05 , it could indicate either a weak association, or that there is insufficient data to support the relationship. The risk of having an alpha error can be minimized by decreasing the acceptable alpha value to 0.01 or less.

If we fail to reject the null hypothesis, we risk a beta error by concluding that there is no difference when in actuality there is one (Norman \& Streiner, 2009). A beta error could be of concern as the test concluded that there was no difference between the knowledge scores of participants with food allergies, with those without food allergies. A contributing factor to this is the small sample size $(\mathrm{N}=17)$ of the group with food allergies. The beta error could potentially be eliminated if the sample size was increased, thereby helping reduce any false conclusions.

Lastly, power is the probability of concluding there was a difference when in fact there was one (Heacock \& Sidhu, 2014). Power is not given for chi-square tests, but the power for the t-test were $0.11947(\alpha=0.05)$ and $0.03415(\alpha=0.01)$. The power given in this test is quite weak, which 
means that the research design may not be sensitive enough to detect any effects (Aberson, 2002).

\section{DISCUSSION}

Public awareness of allergens and food allergies is something that communities should strive towards to develop a healthier and safer community. Presently, people are more allergy alert as members of their family, friends, and even colleagues are vulnerable to it (Vogel, 2015). For instance, small steps have been taken towards effective allergen awareness in communities through initiatives such schools displaying allergen safety signs within their classrooms, and restaurants providing detailed menu ingredients readily available to their customers. Yet these actions may not be sufficient in the large-scale of public health. It is likely that not all individuals attending a particular school, or working at a particular restaurant fully understands what a food allergy is or its relevance in society. According to Ben-Shoshan et al. (2012), studies have suggested an increasing prevalence of food allergies with rates slowly stabilizing in the most recent decade. Unfortunately, even with this knowledge of risk, the behavior of many remains unchanged. Individuals who are not directly influenced by food related health concerns, whether their own or those of someone they know do not, seem not to be bothered with getting educated on the subject matter themselves. By surveying EHOs, this study attempted to determine the extent of their knowledge on food allergens, and whether their knowledge was based on them having a food allergy. The t-test concluded that there was no difference between the knowledge scores of EHOs, regardless of whether or not they had a food allergy. The chi-square test concluded that there was an association between behaviors of the EHOs in terms of the extent of education they provided to food operators, and whether or not they had a food allergy. The conclusion that there was no difference may have been a result of a $\beta$ error, which indicates that there is no difference when in reality there actually is or could be one. It is likely that the small sample size $(\mathrm{N}=17)$ of the group with allergies was a contributing factor producing insignificant conclusions. Additionally, the power given by the test is quite weak, which means that the research design may not be sensitive enough to detect any effects.

Previously conducted research indicated that approximately one in thirteen (7.69\%) people have food allergies (Picard, 2010), but the results were much higher in this study with almost one in four (24.28\%) participants having food allergies. This difference could be a result of the smaller sample population or simply due to chance. Nonetheless, more research should be conducted to obtain a better approximation of the number of individuals living with food allergies both provincially and nationally.

The results of the study did not prove to be significant based on food allergen knowledge, but showed significance when taking into consideration the behaviors and opinions of the participants. For example, to the question "How often do you educate food operators on allergen safety?" more of the participants with food allergies answered "Most of the time" in relation to the other options provided. Being directly affected by food allergies the EHO may be more sensitive to the subject matter, and therefore place more emphasis on it post inspection. That being said, a majority of the EHOs without food allergies responded with "Some of the time" or "A few times/Rarely." The respondents may have answered as so because they may not be as knowledgeable or aware of the subject matter, and therefore, either forget or are less comfortable educating individuals on the subject matter. Additionally, unless allergies are addressed in the food establishment's Hazard Analysis and Critical Control Points (HACCP) Plan, "an internationally recognized, science-based food safety system, designed to prevent, reduce or eliminate potential biological, chemical and physical food safety hazards" there are no other checkpoints or requirements the EHO must monitor and approve during a restaurant inspection (CFIA, 2014a). As a result of this, worries and hazards regarding food allergens are often dismissed.

Furthermore, EHOs without food allergies responded to the opinion questions similarly to EHOs with food allergies, and rated their knowledge level on the subject matter as high. This answer was likely based on perception rather 
than actual knowledge regarding all food allergens. Those who answered in that particular way may have an adequate knowledge of particular allergic proteins and foods they have been exposed to, but potentially not of the priority allergens or of the different forms of food sensitivities one can have.

As previously stated, food allergies are on the rise and people are starting to pay more attention to them. But until allergens are regulated, and food inspectors are able to enforce these regulations, it is likely that the topic of food allergens will not be of primary focus. Currently the one way EHOs can address the topic of allergens is by ensuring that allergens get addressed in the Establishment's HACCP or Food Safety Plan. They can do this by including a list of each menu item that contains any of the ten priority allergens, and making sure that this list is available to all staff as well as to the public on a demand basis.

\section{Recommendations}

The main recommendation would be to continue to increase not only EHO knowledge regarding food allergens and food allergen safety, but the knowledge of the public. This way it is more of a collaborative effort where everyone can be involved in creating a safer society for vulnerable populations. Using relevant literature, in addition to the results obtained through this research, can provide a basis for establishing a food allergen training program for EHOs or food industry specialists in the future. Canadian Food Industries can look to U.S programs such as the online course ServSafe Allergens, as well as the AllerTrain Program, which provides allergen training to chefs and food service employees regarding safe handling procedures of meals (Food Allergy Research \& Education, 2015), to help create a suitable program addressing hazards and concerns commonly dealt within Canada.

Additionally, the Government of Canada can continue to work towards changing the way food packages are labelled. As previously mentioned, Culhane (2012) mentioned how in the future they want food labels in Canada to list the priority food allergen by its official name next to the ingredient. By doing this, allergens of primary concern are highlighted and consumers are made more aware.
In terms of this particular research study, an appropriate recommendation pertaining to the knowledge testing portion of the survey would be to provide an additional option of "I do not know" or "Unsure." If this was implemented it could have potentially helped in determining whether the participant actually knows the answer to the question or if it was a random selection. This ultimately can provide more "true" results, and could potentially change the conclusions made in the analysis stage of the study.

Finally, more nationwide studies are required to get a more up-to-date number of how many people live with food allergies. With more current statistics, public health programs, awareness campaigns, guidelines, and legislation can be formed to better address the problems affecting society.

\section{Limitations}

One of the greatest limitations in the study was that the sample size was small, especially for the group of participants with allergies. Consequently, the results that were obtained may not have been a true representation of the collected allergen knowledge scores. On top of that the data collection period could be longer to gather more data. Communication was a major problem encountered during the data collection period. A lot of advanced notice was required to help distribute the survey to members of CIPHI, and there was no set or standard way to approach the different health authorities. Because of this, there were fewer respondents from out of province and the study was limited to B.C. Although, it was designed to collect data from participants nationally, only three percent of the respondents were from out of province. Therefore, further analyses regarding whether the location of work had any impact on the level of food allergen knowledge could not be determined. Moreover, the methods in which the survey was distributed may not have been effective or efficient. The survey was distributed using two different Facebook groups, various emails to EHOs employed at a couple health authorities, followed by emails to the directors of the B.C. health authorities who were asked to distribute it to their staff. This method resulted in the survey being 
repeatedly sent to a lot of the same people, instead of reaching a broader more diverse audience. This is likely another contributing factor to the lower number of respondents.

Lastly, the research design may have not been sensitive to detect the variances in the two different test groups. Therefore, the survey needs to be altered in some way to help detect significant effects and outcomes. This can be done by limiting the number of options available in the multichotomous ordinal and nominal questions. This in turn makes each response group larger, and potentially resulting in more significant results.

\section{Future Research Suggestions}

For future research, the scope of the study could be broadened to check public knowledge regarding food allergens as opposed to solely EHO knowledge. It was previously mentioned in the recommendation section that not only should EHO knowledge and awareness be raised in terms of food allergens, but public knowledge and awareness should be heightened as well. By minimizing the exclusion criteria, we can possibly achieve this. The ultimate goal is to in some way change public policy to create a society in which people feel safe, and feel as though their health is one of the government's primary interests. The same research study could also be distributed to restaurant operators, and they could be asked whether they would want an AllerTrain type program. If majority of the respondents agree the allergen awareness and safety are major problems in the food industry, and that they would benefit from such a program, maybe the government will put more consideration into establishing courses and training sessions addressing their concerns.

\section{CONCLUSIONS}

The statistical test performed in this study regarding allergen knowledge concluded that the participant's knowledge regarding food allergens was not dependent on the presence or absence of a food allergy, but rather a general awareness of the subject matter. The other test performed regarding EHO behavior, proved that the frequency of food allergen education to restaurant operators during the inspection of their facility was dependent on whether they had a food allergy themselves or not.

There are numerous individuals living with food allergies and food sensitivities, therefore, it is important that industry professionals such as allergists, scientists, the food industry itself, and food regulators including EHOs and Food Inspectors are able to properly identify allergens of concern and make allergen safety one of their top priorities. Food allergies are important, though, it can be said that it is up to the consumer to educate themselves. Many in the food industry may agree public health cannot completely focus on what can be described as 'hypersensitive' individuals, but on the population as a whole. Hopefully this insight can be changed. This study which is based on allergen knowledge and perception is one step towards addressing the deficit that exists with regards to managing food allergens in public health. Health Authorities can use the results to provide a basis for establishing a food allergen training program for EHOs in the future.

\section{ACKNOWLEDGEMENTS}

Thank you to British Columbia Institute of Technology's Environmental Health Department for their support towards this research project. A special thanks to Helen Heacock for providing guidance, encouragement, and her much appreciated enthusiasm during this project.

\section{COMPETING INTEREST}

The authors declare that they have no competing interests.

\section{REFERENCES}

Aberson, C. (2002). Interpreting Null Results: Improving Presentation and Conclusions with Confidence Intervals. Journal of Articles in Support of the Null Hypothesis, 1(3): 36-42.

Anaphylaxis Canada. (2014). Key Facts. Retrieved from http://www.anaphylaxis.ca/en/i_want/anaphylaxis 101/key_facts.html 
Asthma and Allergy Foundation of America. (2005). Chemical Sensitivities: Chemical and Other Environmental Sensitivities. Retrieved from http://www.aafa.org/display.cfm?id=9\&sub=20\&c ont $=282$

Ben-Shoshan, M., Harrington, D.W., Soller, L., Fragapane, J., Joseph, L., St Pierre, Y., Godefroy, S.B., Elliot, S.J., \& Clark, A.E. (2010). A population-based study on peanut, tree nut, fish, shellfish, and sesame allergy prevalence in Canada. American Academy of Allergy, Asthma, \& Immunology, 125(6), 1327-1335. doi:

10.1016/j/jaci.2010.03.015

Ben-Shoshan, M., Harrington, D.W., Soller, L., Fragapane, J., Joseph, L., St Pierre, Y., Godefroy, S.B., Elliot, S.J., \& Clark, A.E. (2012).

Demographic Predictors of Peanut, Tree Nut, Fish, Shellfish, and Sesame Allergy in Canada. Journal of Allergy, 2012, 1-6. doi:10.1155/2012/858306

Canadian Food Inspection Agency. (2011). Government of Canada Improves Access to Allergen Information. Retrieved from http://www.inspection.gc.ca/about-thecfia/newsroom/news-releases/allergeninformation/eng/1323652435058/1323652435059

Canadian Food Inspection Agency. (2013). Statistics: Food Recall Incidents. Retrieved from http://www.inspection.gc.ca/about-thecfia/newsroom/food-safety-system/food-recallsincidents/eng/1348756225655/1348756345745

Canadian Food Inspection Agency. (2014a). Food Safety Enhancement Program Manual. Retrieved from http://www.inspection.gc.ca/food/safe-foodproduction-systems/food-safety-enhancementprogram/programmanual/eng/1345821469459/1345821716482?cha $\mathrm{p}=2 \# \mathrm{~s} 2 \mathrm{c} 2$

Canadian Food Inspection Agency. (2014b). List of Ingredients and Allergens: Definitions.

Retrieved from http://www.inspection.gc.ca/food/labelling/foodlabelling-for-industry/list-of-ingredientsandallergens/eng/1383612857522/1383612932341 ?chap=5\#s19c5
Culhane, C.T. (2012). Food Allergen Labelling in Canada. Retrieved from http://www.ohea.on.ca/uploads/1/2/6/0/12605917/ food_allergen_labelling_in_canada.pdf

Food Allergy Research \& Education. (2015). Tools \& Resources: Training Programs. Retrieved from http://www.foodallergy.org/trainingprograms

Google Docs. (2014). Retrieved from docs.google.com

Government of Canada. (2012). Mustard: One of the ten priority food allergens. Retrieved from http://www.hc-sc.gc.ca/fnan/alt_formats/pdf/pubs/securit/2012allergen_mustard-moutarde/2012allergen_mustard-moutarde-eng.pdf.

Government of Canada. (2013). Safe school lunches. Retrieved from http://healthycanadians.gc.ca/eatingnutrition/safety-salubrite/school-lunch_repasecole-eng.php

Gupta, R.S., Kim, J.S., Springston, E.E., Pongracic, J.A., Wang, X., \& Holl, J. (2009). The Chicago Food Allergy Research Survey for the General Public. Retrieved from http://www.biomedcentral.com/content/supplemen tary/1472-6963-9-142-S3.pdf

Harvard Health Publications. (2011, May) Food allergies and food intolerances. Both are on the rise - and it's important to know the differences. Harvard Women's Health Watch, 18(19), 4-6.

Heacock, H. \& Sidhu, B. (2014). ENVH 8400 Power point lecture slides. British Columbia Institute of Technology, Burnaby, BC.

Health Canada. (2009). Food Allergies. Retrieved from http://www.hc-sc.gc.ca/hl-vs/iyh-vsv/foodaliment/allerg-eng.php

Health Canada. (2012). Food Allergies and Intolerances. Retrieved from http://www.hcsc.gc.ca/fn-an/securit/allerg/index-eng.php

Hintz, J. (2013). NCSS 9. NCSS, LLC. Kaysville, 
Utah USA. www.ncss.com

Lee, E.T. \& Wang, J.W. (2013). Statistical Methods for Survival Data Analysis. Hoboken, NJ: Wiley.

Lucas, J.S.A. \& Atkinson, R.G. (2008). What is a food allergen? Clinical and Experimental Allergy, 38: 1095-1099. doi: 10.1111/j.13652222.2008.02988.x

McHugh, M.L. (2013) The Chi-square test of independence. Biochemia Medica, 23(2): 143-149. doi: 10.11613/BM.2013.018

Microsoft Corporation. (2010). Microsoft @ Excel () 2010.

National Institute of Allergy and Infectious Diseases. (2010). What is Food Allergy? Retrieved fromhttp://www.niaid.nih.gov/topics/foodAllergy/ understanding/Pages/allergicRxn.aspx

Norman, G.R. \& Streiner, D.L. (2009). Power tripping: alpha errors, beta errors and power. Community Oncology, 6(7): 322-327.

Picard, A. (2010, September 20). One in 13 Canadians has serious food allergy. The Globe and Mail. Retrieved from http://www.theglobeandmail.com/life/health-andfitness/one-in-13-canadians-has-serious-foodallergy/article599131/

Reliability. (2014). In Oxford English Dictionary. Retrieved from Oxford English Dictionary. Retrieved from http://www.oed.com.proxy.lib.sfu.ca/view/Entry/1 61904?redirectedFrom=reliability\#eid

Sampson, H. A. (2004). Update on food allergy. Journal of Allergy and Clinical Immunology, 113(5): 805-819. doi: 10.1016/j.jaci.2004.03.014

Sicherer, S.H. (2011).Epidemiology of food allergy. Journal of Allergy and Clinical Immunology, 127(3): 594-602. doi:10.1016/j.jaci.2010.11.044

Soller, L., Ben-Shoshan, M., Harrington, D.W., Fragapane, J., Joseph, L., St Pierre, Y., Godefroy,
S.B., La Vieille, S., Elliot, S.J., \& Clarke, A.E. (2012). Overall Prevalence of Self-reported Food Allergy in Canada. Journal of Allergy and Clinical Immunology,130(4): 986-988. doi:

10.1016/j.jaci.2012.06.029

SurveyMonkey. 2015. SurveyMonkey. Retrieved from https://www.surveymonkey.com/

U.S. Food and Drug Administration. (2013).

Retrieved from

http://www.fda.gov/Food/GuidanceRegulation/Gui danceDocumentsRegulatoryInformation/Allergens /ucm106108.htm

University of Nebraska - Lincoln. (2014).

Allergenic Foods and their Allergens, with links to Informall. Retrieved from

http://farrp.unl.edu/informallbig8

Vogel, C. (2015, Mar 1). Families learn to cope with food allergies as awareness, support increase. The Buffalo News. Retrieved from http://www.buffalonews.com/cityregion/medical/families-learn-to-cope-with-foodallergies-as-awareness-support-increase-2015030 
